\section{How much do you want to know?}

\section{Joseph Ford}

Hamiltonian Dynamical Systems. Compiled and introduced by $\mathrm{R}$. S. MacKay and J. D. Meiss. Adam Hilger, BristollTaylor \& Francis, Philadelphia: 1987. Pp. 784. Hbk £49.50, \$109; pbk £20, $\$ 44$.

Creators of reprint anthologies appear to have no thought for the morrow, theirs or ours, for the product of their enthusiasm sweeps us all into a no-win/no-lose situation. Who can resist buying such a magnificent potpourri of reprint classics? Yet, who would wish to clutter his private library with such a mass of accompanying filler material? And thus our quandary grows: how many the classics, how numerous the fillers? Towards which side does the balance tip? Do the compilers know; do reviewers?

Consider the compilers. After diligently searching their memories and their reprint stacks for papers that deeply influenced their own research, they compose a table of contents, and in the process succumb to the temptation to add a few of their own articles to the list. Consultation with friends and colleagues fills in obvious gaps and extends horizons until severe dieting is required to bring the size of the book down to 800 pages. They then at last present us with a compilation which most assuredly includes many masterpieces; yet should not many of the included articles have been left out and vice versa? On judgemental issues of such delicacy, should or even can we trust anthologists not yet grey at the temples?

Consider the reviewers. The present reviewer, a physicist by trade, has favourably reviewed Chaos, an earlier reprint anthology published by World Scientific in 1984, and edited by the physicist, Hao Bai-Lin. Philip Holmes, a distinguished theoretical mechanician at Cornell, has written a scathing review of this same anthology. In particular, he deplores the physicist's myopia and the lack of appreciation for rigour that is reflected in Hao Bai-Lin's selection of reprints. Turning to Hamiltonian Dynamical Systems, one feels confident that Professor Holmes would rejoice in the extensive mathematical coverage of its subject area, whereas I am overwhelmed by its welter of formal articles which tell you much more than you wanted to know about mathematically rigorous developments. Given this consistent disagreement between tribal elders regarding the worth of anthologies, should or even can we trust such delicate judgemental issues to reviewers, no matter how grey they are at the temples?
If neither reviewers nor compilers suffice, how is a judgement to be made? Alas, in the face of such ambiguity, each buyer must reach his own decision; nonetheless, perhaps a few helpful hints can be provided.

At the outset, it must be emphasized that this anthology is strictly a reference work. Experts who read its table of contents, and find they know or wish to know most of the articles, should seriously consider the convenience of owning the book. Those less familiar with its contents should be wary, however, for many of the articles are highly mathematical and may prove quite opaque to all but the dedicated specialist. Moreover, there is little introductory or explanatory material to guide either the novice or the expert. Nonetheless, provision of the remarkably lucid, now classic review papers by Berry, by Moser, and by Arnol'd in the same volume with the seminal paper on Arnol'd diffusion, Chirikov's pioneering and almost readable paper on resonance overlap, John Greene's inventive but sometimes cryptic paper clarifying the notion of a transition to chaos, and Jack Wisdom and colleagues' charming paper on chaos in the Solar System is strong prima facie evidence that this book is worth the price.

And yet not everyone may wish to pay this price, because many do not belong to the audience to whom the volume is addressed. Recall that scientists tend to fall into two categories: those who accept without hesitation the notion that a simple closed curve divides the plane into an inside and an outside and those who do not. Humiltonian Dynamical Systems is a volume created for those who do not! Once this determined bias towards rigour is recognized, the buyer is at liberty to accept or reject the volume.

There is, however, a much more serious

\section{Firm foundations}

\section{David Oldroyd}

From Mineralogy To Geology: The Foundations of a Science, 1650-1830. By Rachel Laudan. University of Chicago Press:1987. Pp.278. \$27.50, £21.95.

This volume is an invaluable addition to the small number of books in English on the early history of gec,logy. Although, as Laudan's excellent bibliography shows, there is now an extensive secondary literature on the topic, there have been few attempts at synthesis of this material by authors who know the literature intimately and who have themselves contributed to it.

Geologists interested in the early history of their subject will probably be chiefly familiar with Adams's Birth and and yet much less obvious defect in this work, which lies in its presentation of a one-sided view of Hamiltonian dynamics. Its pages discuss quite remarkable progress, but always evolutionary progress in which today appears as little more than a magnified image of yesterday. Yet computational complexity theory, algorithmic complexity theory and cellular automata theory have long since established that chaos is defining limits on man's abilities no less revolutionary than those implied by $h$ and $c$, and that Gödel, Turing, Church and Post are as relevant to Hamiltonian dynamics as they are to mathematical logic.

Although I cannot here even summarize all the issues involved, perhaps a simple question may provide at least the flavour of the thing. Existence-uniqueness theorems notwithstanding, how are we to assign a physical or a computational meaning to the notion of a chaotic orbit which not only has exponential sensitivity to slight inaccuracies in its initial state but which is also indistinguishable from a realization of some random process? A preliminary and partial 'practical' answer is provided by the Anosov-Bowen $\beta$-shadow lemma which, remarkably enough, does not appear to be discussed in this anthology; regardless, the logical issue remains unresolved because a chaotic (random) orbit contains more information than all of man's logical systems combined. How then are we to define the indefinable, compute the uncomputable?

MacKay and Meiss have in all truth compiled an excellent anthology for those who have developed a tolerance for being told both more and less than they wanted to know.

Joseph Ford is a Regents' Professor in the School of Physics, Georgia Institute of Technology, Atlanta, Georgia 30332, USA.

Development of the Geological Sciences (1938) and Geikie's Founders of Geology (1905/1962). For Adams, study of early geology revealed the quaint ideas of our forebears. For Geikie, it demonstrated particularly the soundness of reasoning and method of early British (especially Scottish) geologists in comparison with their German contemporaries. Now Laudan skilfully displays the theoretical underpinnings of early geology, studiously avoiding any sense of disdain towards outmoded theories, or partisan leanings, and argues that the main framework for geological research in the early nineteenth century was set by the Wernerian School of Freiberg, rather than the Huttonians of Edinburgh. Geologists may also be interested, and perhaps surprised, to learn that Smith did not always practise what he preached. Although he undoubtedly enunciated and used the stratigraphical principle with which his 